\title{
Management of hospitalized type 2 diabetes mellitus patients
}

\author{
Juan José Marín-Peñalver, Iciar Martín-Timón, Francisco Javier del Cañizo-Gómez \\ Section of Endocrinology, University Hospital Infanta Leonor, School of Medicine, \\ Complutense University, Madrid 28031, Spain
}

\begin{abstract}
Both hyperglycemia and hypoglycemia in hospitalized patients are associated with adverse outcomes including increased rates of infection, longer hospital length of stay, and even death. Clinical trials in patients with type 2 diabetes mellitus proved that by improving glycemic control, we can reduce all of them. Insulin is the preferred treatment for glycemic control in most cases, but alternative treatment options that can normalize blood glucose levels without hypoglycemia are being sought. Moreover, hospitalized patients are particularly vulnerable to severe, prolonged hypoglycemia since they may be unable to sense or respond to the early warning signs and symptoms of low blood glucose. Finally, nutritional support, corticosteroid therapy, and surgery increase the risk of hyperglycemia that leads to an increased risk of morbidity and mortality. We review the management of type 2 diabetes mellitus patients who are admitted to the general medical wards of the hospital for a procedure of intercurrent illness.
\end{abstract}

Key words: type 2 diabetes mellitus, in-hospital diabetes control, incretins, hyperglycemia, hypoglycemia, nutritional support, corticosteroids, surgery

\section{INTRODUCTION}

Both hyperglycemia and hypoglycemia in hospitalized patients are associated with adverse outcomes including increased rates of infection, longer hospital length of stay, and even death. ${ }^{[1,2]}$ Acute illness results in a number of physiological changes, such as increases in circulating concentrations of stress hormones, or therapeutic choices, such as glucocorticoid use that can exacerbate hyperglycemia. Hyperglycemia, in turn, causes physiological changes, such as decreased immune function and increased oxidative stress, which can exacerbate acute illness. This results in a vicious cycle of uncontrolled blood sugar and worsening of the disease..$^{[3]}$

Randomized clinical trials in no critically and critically ill patients with type 2 diabetes mellitus (T2DM) proved that by improving glycemic control, we could reduce all of them. Consequently, the hospital objectives for T2DM patients must include improved glycemic control (preventing hypo- and hyperglycemia) so that they can reduce hospital complications, systemic infections, hospital stay, and hospitalization cost and provide an effective transition out of hospital so as to prevent readmission.

Here, we review the management of T2DM patients who are admitted to the general medical wards of the hospital for a procedure of intercurrent illness. The treatment of hyperglycemia in patients with artificial nutrition and with corticosteroid therapies, and the perioperative management of T2DM are also discussed.

\section{CONSIDERATIONS ON ADMISSION}

We must measure glucose level in all patients admitted to the hospital as is advised in clinical guidelines. ${ }^{[2,4]}$ Those with hyperglycemia, defined as blood glucose $>140 \mathrm{mg} / \mathrm{dL}$, and those with a history of T2DM should be monitored with glucose testing before meals and at bedtime. If the patient is taking nothing by mouth, testing is recommended every $4-6$ hours. In diabetic patients, HbA1c should be placed if it has 
not been made within the prior 3 months. If we find a "non diabetic" patient with HbA1c $>6.5 \%$, it suggests that diabetes preceded the hospitalization.

\section{GLYCEMIC TARGETS IN HOSPITALIZED PATIENTS}

In hospitalized patients, hyperglycemia is defined as blood glucose $>140 \mathrm{mg} / \mathrm{dL}$.

In critical care settings, a glucose target of $140-180 \mathrm{mg} / \mathrm{dL}$ is recommended for most critically ill patients. ${ }^{[2,4]}$ Data from trials, ${ }^{[5,6]}$ using intensive glycemic control in patients in intensive care unit (ICU) failed to show a significant improvement in mortality and in some cases, showed an augmented mortality risk. The GLUCO-CABG trial, ${ }^{[7]}$ in patients after cardiac surgery also demonstrated no differences in rates of complications and death between an intensive glucose management of $100-140 \mathrm{mg} / \mathrm{dL}$ and a traditional target of $140-180 \mathrm{mg} / \mathrm{dL}$. However, more stringent objectives as $110-140 \mathrm{mg} / \mathrm{dL}$ may be appropriate for select patients if they are achievable without meaningful risk of hypoglycemia, for example, patients with acute myocardial infarction ${ }^{[8]}$ or neurological events.

In non-critical care settings, a glucose target between 140 and $180 \mathrm{mg} / \mathrm{dL}$ is recommended. Higher glucose levels may be adequate in terminally ill patients or with severe comorbidities. ${ }^{[4]}$ An acceptable goal to prevent hypoglycemia is to attain pre-meal blood glucose no lower than $90-100 \mathrm{mg} / \mathrm{dL}$.

\section{INPATIENT MANAGEMENT ${ }^{[9]}$}

\section{Insulin}

In critical care setting, continuous intravenous insulin infusion is the preferred way to control hyperglycemia. ${ }^{[2]}$ The administration should be based on validated protocols. The glucose measurements should be made every hour until stable glycemic control is achieved.
In non-critical care setting, basal insulin is administered once or twice daily in combination with prandial and correction insulin is the preferred treatment for patients with good nutritional intake. The only use of sliding-scale insulin is strongly dejected. If oral intake is poor, we can administer the short-acting insulin after the patient eats to avoid hypoglycemia. If the patient is taking nothing oral, we can use a basal plus correction insulin regimen.

\section{Alternative treatment options}

The use of oral antidiabetic treatments during the hospitalization is generally not recommended because of the limited data available on their safety and efficacy, and also due their major risk of hypoglycemia and contraindications. Alternative treatment options (Table 1) that can normalize blood glucose levels without undue hypoglycemia are being sought. Incretin-based therapies, such as glucagon-like peptide-1 receptor agonists (RA-GLP1) and dipeptidyl peptidase-4 inhibitors (iDPP-4), may have this potential by increasing glucose-dependent insulin secretion and inhibiting glucagon secretion with a low risk of hypoglycemia. Incretinbased therapies also can be used in combination with insulin to provide further flexibility for blood glucose lowering than what can be obtained by incretins alone.

A recent randomized trial ${ }^{[10]}$ in $\mathrm{T} 2 \mathrm{DM}$ patients treated at home with oral antidiabetics or a low dose of insulin $(<0.4 \mathrm{IU} / \mathrm{kg} /$ day $)$ showed that sitagliptin, alone or in combination with basal insulin resulted in similar glucose control, frequency of hypoglycemia, and length of hospitalization compared with a basal bolus insulin regimen. The sitagliptin groups required a lower total daily dose of insulin and fewer insulin injections. A large multicenter trial aimed to determine the safety and efficacy of sitagliptin therapy for in-hospital and post-discharge management of general medicine and surgical patients with T2DM, is currently ongoing. ${ }^{[11]}$

Several trials have evaluated RA-GLP1 infusion therapy, alone or in combination with insulin, in diabetic ICU

\begin{tabular}{lllll}
\hline \multicolumn{7}{l}{ Table 1. Antihyperglycemic agents in the hospital setting } & \\
\hline & Experience & Risk of hypoglycemia & Glucose-lowering effect & Posible adverse effect \\
\hline Insulin & Extensive & + & ++ & Errors in administration \\
Metformin & Limited & - & ++ & Lactic acidosis \\
& & & & Gastrointestinal \\
SFO & Limited & + & ++ & Risk of hypoglycemia \\
RA-GLP1 & Limited & - & ++ & Gastrointestinal \\
iDDP-4 & Limited & - & + & Contraindicated in pancreatitis \\
iSGLT2 & Limited & - & ++ & Risk of urinary and genital infections \\
& & & & and hypotension \\
\hline
\end{tabular}

SFO: Sulfonylureas; RA-GLP1: glucagón-like peptide-1 receptor agonists; iDPP-4: dipeptydyl peptidase-4 inhibitors; iSGLT-2: sodium-glucose cotransporter-2 inhibitors 
patients. ${ }^{[12-15]}$ In T2DM patients with oral agents, with mild to moderate insulin resistance, glucose control was successful with incretins alone, but insulin was required in those with significant insulin resistance. RA-GLP1 use decreased the cumulative insulin dose, the frequency of insulin titrations, the time to reach steady state glucose and glucose variability. ${ }^{[12-15]}$

Incretin therapies show promise in inpatient management. ${ }^{[16]}$ DeFronzo and Schwartz have described a protocol to use incretins in hospital admission and at discharge. ${ }^{[17]}$ They suggest starting with incretins, before or immediately after admission to the hospital, to prevent hyperglycemia in most diabetic patients. Insulin should be added in the case of persistent hyperglycemia, initially with correction scale, later changing to basal insulin if necessary. They also suggest that incretins be continued until discharge.

\section{Treatment of hypoglycemia}

Hospitalized patients are particularly vulnerable to severe, prolonged hypoglycemia since they may be unable to sense or respond to the early warning signs and symptoms of low blood glucose. Hypoglycemia is defined as blood glucose $<70 \mathrm{mg} / \mathrm{dL}$. Severe hypoglycemia occurs when glucose is $<40 \mathrm{mg} / \mathrm{dL}$. The prevalence of hypoglycemia in critically ill patients ranged between $5 \%$ and $28 \%,{ }^{[18]}$ and in noncritical care settings between $1 \%$ and $33 \% .{ }^{[19]}$

The most common risk factors are older age, change in nutritional intake (reduced oral intake, emesis, unexpected interruption of enteral or parenteral feedings), inappropriate administration of short-acting insulin's relation to meals and failure to adjust treatment when we reduce the infusion rate of intravenous dextrose or steroid therapy. ${ }^{[20,21]}$

Hypoglycemia in hospitalized T2DM patients has been associated with poor outcomes and 2.8 days longer hospital stay. ${ }^{[22]}$ However, other studies suggest that the increased mortality rate associated with hypoglycemia is only with spontaneous hypoglycemia rather than drug-associated hypoglycemia. ${ }^{[23]}$ The causes of hypoglycemia in hospitalized T2DM patients include continued hypoglycemic therapy, such as sulfonylureas, when caloric intake is stopped or reduced, use of standard sliding-scale insulin inadequately, ${ }^{[2]}$ errors in insulin dosing, and implementation of intensive insulin therapy in critically ill patients. ${ }^{[25]}$

Prolonged QT interval, ischemic electrocardiogram changes, arrhythmias, and sudden death are also associated with hypoglycemia. ${ }^{[2]}$ Inpatient mortality was significantly higher for patients with at least one hypoglycemic $(<50 \mathrm{mg} / \mathrm{dL})$ episode. ${ }^{[22]}$
We can prevent or reduce hypoglycemic events in hospitalized patients by: ${ }^{[27]}$

- Recognizing precipitating factors.

- Ordering appropriate scheduled insulin or antidiabetic oral agents.

- Monitoring blood glucose at the bedside.

- Educating patients, family, friends, and staff about symptom recognition, and appropriate treatment.

- Providing appropriate nutritional requirements.

- Applying systems for eliminating or reducing medication and treatment errors.

For the treatment of hypoglycemia, there should be a standardized protocol to immediately tackle hypoglycemia. If the patient is conscious and can be safely treated with oral carbohydrate, use an appropriate choice of liquid or easily dissolved glucose tablets. If the patient is unconscious, then intravenous access for quick administration of dextrose or intramuscular injection of glucagon are the preferred treatment methods. After treating a hypoglycemic event, search for the cause, correct the problem, and, if indicated, modify treatment.

\section{Nutritional support}

Nutritional support increases the risk of hyperglycemia that leads to an increased risk of morbidity and mortality. However, there is little literature available with specific recommendations for this situation. ${ }^{[28]}$

Enteral nutrition: Enteral nutrition increases the risk of hyperglycemia in hospitalized T2DM patients. Before beginning enteral nutrition, it is important to know the metabolic status, previous control of diabetes, and insulin requirements. ${ }^{[2]]}$

Continuous enteral nutrition has an effect, not totally known, in secretion and action on incretin hormones and can contribute to hyperglycemia. Hospitalization produces insulin resistance and together with delivery of glucose and gluconeogenic substrates via enteral contribute to hyperglycemia. ${ }^{28,30]}$

Hyperglycemia can be treated with the use of diabeticspecific formulas and different insulin regimens. Although some institutions do not have recommendations about this, ${ }^{[31]}$ diabetic-specific formulas have been suggested in patients with persistent hyperglycemia. ${ }^{[29]}$

Retrospective studies with different insulin regimens have demonstrated effectiveness to manage hyperglycemia with a variable degree of control. These studies use basal insulin glargine once daily, NPH every 4 or 6 hours, or $70 / 30$ biphasic insulin twice or 3 times a day. ${ }^{[32-34]}$ Another approach is to use sliding-scale regular insulin regimens. ${ }^{[28]}$ 
Nevertheless, there are also concerns with respect to the use of long-acting insulin causing hypoglycemia with interruption of nutrition; for this reason, some institutions prefer to use only short-acting insulin or intermediate-acting insulin. ${ }^{[28]}$ However, there is only a prospective study that has compared the effectiveness and risk of hypoglycemia between basal insulin versus sliding-scale regular insulin, and showed that use of sliding-scale regular insulin is not sufficient in most diabetics and requires addition of NPH insulin. For that, the early addition of glargine or NPH insulin is secure and useful, and it is preferred over slidingscale regular insulin isolated. ${ }^{[35]}$

There are a lot of strategies in insulin treatment for managing hyperglycemia in patients with enteral nutrition and it is important to individualize each case keeping in mind the type and amount of nutrition, previous and recent blood glucose control. Sliding-scale regular insulin can be an initial option in some patients, ${ }^{[28]}$ but the addition of basal insulin is necessary in the majority of diabetic patients. ${ }^{[35]}$

In conclusion, with continuous enteral nutrition, a good management technique can be to administer a basal insulin once daily (glargine, determir) or twice a day (determir/ NPH) together with a rapid-acting insulin in divided dose every 4 hours or preferably, for less injections, a regular insulin divided dose every 6 hours. If the continuous enteral is cyclical, we also can administer a basal insulin at the time of starting enteral nutrition and a combination of rapidacting or regular insulin only during the time of nutrition, and administer before 4 or 6 hours finish the nutrition. In case of bolus feeding, the best approach can be to administer short- or rapid-acting insulin before each bolus. ${ }^{[4,36]}$

Parenteral nutrition: Hyperglycemia is a common metabolic complication of parenteral nutrition and it involves unsatisfactory clinical outcomes. Hyperglycemia is caused by excess of parenteral glucose and metabolic changes occurring in hospitalized patients as a result of the hospitalization stress; this produces mediators and interferes with carbohydrate metabolism producing more hepatic gluconeogenesis and less glucose utilization in peripheral tissues. It is important to control blood glucose every 6 hours after parenteral starting nutrition. ${ }^{[30,37]}$

Subcutaneous and intravenous insulins are valid options for managing hyperglycemia. The use of intravenous infusion of insulin is reserved for critical patients where exist the possibility for a tighter control, ${ }^{[30,38]}$ and patients with marked hyperglycemia because is achieves better control. ${ }^{[4,39]}$ For more stable patients and patients in non-ICU, the insulin can be administered by mixing it in nutritional parenteral bag safely. ${ }^{[30]}$ When patients are initially treated with intravenous insulin infusion, we can estimate the dose in parenteral nutrition bag. ${ }^{[4]}$ Other option is adding insulin in the ratio of 1 unit of insulin per 10 or 11 grams of dextrose in patients receiving 150-300 grams of carbohydrates per day. ${ }^{[30]}$ Subcutaneous correction dose of regular insulin must be used to correct hyperglycemia and regulate the dose of insulin. ${ }^{[4]}$

The amount of insulin depends on clinical situation, quantity of carbohydrates, and metabolic status. One study shows the relationship between requirements of insulin in T2DM patients with parenteral nutrition and different predictors, with a heavy relationship with ICU admission, overweight or obesity, and blood glucose control $>120 \mathrm{mg} / \mathrm{dL}$ on day, that parenteral nutrition initiate or a mean $>$ of $180 \mathrm{mg} / \mathrm{dL}$ during receiving the nutritional support. ${ }^{[40]}$

There are not many articles that study the administration of glargine in patients with parenteral nutrition and these studies have different results about glycemic control. Moreover, if parenteral nutrition is stopped, the long action of glargine can produce hypoglycemia and it is important to use the adequate mechanism to avoid this. For this, there is not a firm conclusion about the use of this insulin in patients with parenteral nutrition. ${ }^{[41,42]}$

\section{Corticosteroid therapies}

Hyperglycemia is a frequent complication in patients with corticosteroids. It is prudent monitoring blood glucose at least 48 hours in patients with high dose of them, ${ }^{[2]}$ and it can be stopped in non diabetic patients when the blood glucose control is under $140 \mathrm{mg} / \mathrm{dL}$ without treatment and corticosteroids dose is not going to raise. The mechanism whereby hyperglycemia occurs is increased hepatic gluconeogenesis and a diminished glucose uptake in peripheral tissues. The principal risks factors for hyperglycemia in patients with T2DM are body mass index, age and high dose of corticosteroids. ${ }^{[4,43]}$

Insulin therapy is the elected treatment. Most patients with glucocorticoids may be managed with a subcutaneous basal bolus insulin regimen, ${ }^{[44]}$ with a starting dosage of $0.3-0.5 \mathrm{IU} / \mathrm{kg} /$ day, where $50 \%$ of total daily dose is administered as basal insulin and another $50 \%$ is administered divided in three doses of nutritional insulin. ${ }^{[4,45]}$ One retrospective study suggests that generally, patients need more insulin to achieve normoglycemia, approximately $0.8 \mathrm{IU} / \mathrm{kg} /$ day and proposes give more nutritional insulin: $65-70 \%$ versus $30-35 \%$ of basal insulin. ${ }^{[45]}$ In any case, the starting dosage depends on each patient, keeping in mind the clinical situation, severity of hyperglycemia, and dose of corticosteroids. ${ }^{[4]}$ If patients are previously treated with insulin, it is difficult to predict the new dose, but we must expect an increase in insulin requirements. ${ }^{[43]}$ 
Moreover, correct doses of regular insulin must be prescribed with scheduled insulin. ${ }^{[43]}$ It is necessary to adjust insulin with glucose control, and when there is change in dose of glucocorticoids.

In patients with uncontrolled and severe hyperglycemia and in patients in ICU, an intravenous insulin infusion is more recommendable because it is most efficient in getting normoglycemia. ${ }^{[2,4]}$

Various articles compare NPH insulin with glargine insulin as basal insulin in patients receiving corticosteroid therapy. Both studies show similar effectiveness in the management of hyperglycemia. Although without statistically significat difference, in both articles was found more rate of hypoglycemia in NPH group. ${ }^{[46,47]}$

\section{Surgery}

Hyperglycemia is associated with increased risk for adverse outcomes in patients undergoing surgery: more length of stay, postoperative complications, infections, and more mortality. ${ }^{[48,49]}$

The recommendations and the treatment depend on the type of diabetes, surgical procedure, when patient is going to resume feeding, previous diabetic treatment, glucose control, and metabolic state of patients. ${ }^{[4,50]}$

T2DM patients controlled with diet and exercise, may not need a special preoperative intervention and the glucose can be regulated with correctional regular insulin. Patients with oral antidiabetic or non-insulin injected antidiabetic therapy must discontinue their medications at least 24 hours before surgery and change it for insulin treatment if it is necessary, to achieve normoglycemia. If the patients were treated with insulin previously, they will need insulin in the perioperative period. ${ }^{[4,51]}$

Because basal insulin is for period between meals, on the day of surgery, it must be administered even if the patients are not going to eat. Moreover, we should prescribe a correctional dose of regular or rapid-acting insulin. ${ }^{[4]}$ For long-acting insulin, it is recommended to administer a $75-100 \%$ of morning dose. In case of intermediate-acting insulins, as they have a peak effect, it is suggested to give only $50-75 \%$ of morning dose. For fixed combinations insulins are important consider only the administration of NPH component. In some situations, such as patients with difficult control, intravenous insulin infusion is a good option, although it depends on the accessibility and possibility of patients monitoring. ${ }^{[0,51]}$ Postoperatively, basal bolus regimen is better than sliding-scale insulin to control glucose. If patients do not eat, we must only prescribe basal insulin with a correctional dose of regular or rapid-acting insulin; when patients start to eat, we must administer basal insulin plus nutritional insulin in the meals plus correctional dose. ${ }^{[52]}$

\section{TRANSITION FROM HOSPITAL TO HOME}

During hospitalization, it is important to evaluate and get information concerning the patients' diabetic history: treatment, level of glycemic control, hypoglycemias, $\mathrm{HbA} 1 \mathrm{c}$, and general information about the patient as cultural context, financial resources, and cognitive abilities. This information is useful for deciding if we must to keep or change the treatment when patients come back home. On the other hand, hospitalization can be a good opportunity to review concepts around diabetes with the patients..$^{[2]}$

If $\mathrm{HbA} 1 \mathrm{c}$ is $<7 \%$, patients can be continued with their same outpatient regimen if it is not contraindicated. For patients with $\mathrm{HbA1c}>7 \%$, it is necessary to intensify outpatient diabetic treatment with oral agents, insulin or in combination. In patients with elevated HbA1c and symptomatic or severe hyperglycemia, it is necessary to begin with insulin therapy. We must begin insulin at least 1 day before hospital discharge to check the safety and effectiveness of treatment. ${ }^{[4]}$

It is very important to give patients clear and written information. We must explain and warn about selfmanagement as treatment dosage and timing of administration, hyperglycemia and hypoglycemia, eating patterns, glucose control, and special situations. This is especially important in patients with new medications, insulin regimens, and more complex treatment. ${ }^{[4,9]}$

\section{CONCLUSIONS}

High blood glucose levels in hospitalized patients with T2DM are associated with increased risk of morbidity and mortality. Improved glucose control with insulin injections may improve clinical outcome and prevent some of the hospital complications. Recently, new oral agents as incretins show promise in inpatient management because, alone or in combination with basal insulin, resulted in similar glucose control, and less frequency of hypoglycemia compared with a basal bolus insulin regimen. Other situations as nutritional support, corticosteroid therapies, and surgery should be considered when establishing treatment because of worsening hyperglycemia.

\section{Conflict of Interest}

No conflict of interest. 


\section{REFERENCES}

1. Clement S, Braithwaite SS, Magee MF, Ahmann A, Smith EP, Schafer RG, et al. Management of diabetes and hyperglycemia in hospitals. Diabetes Care 2004;27:553-91.

2. Moghissi ES, Korytkowski MT, DiNardo M, Einhorn D, Hellman R, Hirsch IB, et al. American Association of Clinical Endocrinologists and American Diabetes Association consensus statement on inpatient glycemic control. Diabetes Care 2009;32:1119-31.

3. Inzucci SE. Clinical practice. Management of the hyperglycemia in the hospital setting. N Engl J Med 2006;355:1903-11.

4. Umpierrez GE, Hellman R, Korytkowski MT, Kosiborod M, Maynard GA, Montori VM, et al. Management of hyperglycemia in hospitalized patients in non-critical care setting: an endocrine society clinical practice guideline. J Clin Endocrinol Metab 2012;97:16-38.

5. NICE-SUGAR Study Investigators, Finfer S, Chittock DR, Su SY, Blair D, Foster D, et al. Intensive versus conventional glucose control in critically ill patients. N Engl J Med 2009;360:1283-97.

6. Griesdale DE, de Souza RJ, van Dam RM, Heyland DK, Cook DJ, Malhotra $\mathrm{A}$, et al. Intensive insulin therapy and mortality among critically ill patients: a meta-analysis including NICE-SUGAR study data. CMAJ 2009;180:821-7.

7. Umpierrez G, Cardona S, Pasquel F, Jacobs S, Peng L, Unigwe M, et al. Randomized controlled trial of intensive versus conservative glucose control in patients undergoing coronary artery bypass graft surgery: GLUCO-CABG Trial. Diabetes Care 2015;38:1665-72.

8. Task Force on the management of ST-segment elevation acute myocardial infarction of the European Society of Cardiology (ESC), Steg PG, James SK, Atar D, Badano LP, Blömstrom-Lundqvist C, et al. ESC Guidelines for the management of acute myocardial infarction in patients presenting with ST-segment elevation. Eur Heart J 2012;33:2569-619.

9. American Diabetes Association. Diabetes Care in the Hospital. Diabetes Care 2016;39 Suppl 1:S99-104.

10. Umpierrez GE, Gianchandani R, Smiley D, Jacobs S, Wesorick DH, Newton C, et al. Safety and efficacy of sitagliptin therapy for the inpatient management of general medicine and surgery patients with type 2 diabetes: a pilot, randomized, controlled study. Diabetes Care 2013;36:3430-5.

11. Umpierrez GE, Gianchandani R, Smiley D, Jacobs S, Wesorick DH, Newton C, et al. Randomized controlled trial on the safety and efficacy of sitagliptin therapy for the inpatient management of general medicine and surgery patients with type 2 diabetes: a pilot. Diabetes Care 2013;36:3430-5.

12. Abuannadi M, Kosiborod M, Riggs L, House JA, Hamburg MS, Kennedy KF, et al. Management of hyperglycemia with the administration of intravenous exenatide to patients in the cardiac intensive care unit. Endocr Pract 2013;19:81-90.

13. Mecott GA, Herndon DN, Kulp GA, Brooks NC, Al-Mousawi AM, Kraft $\mathrm{R}$, et al. The use of exenatide in severely burned pediatric patients. Crit Care 2010;14:R153.

14. Marso S, Al-Amoodi M, Riggs L, House J, Peterman D, Kennedy K, et al. Administration of Intravenous Exenatide to Patients with Sustained Hyperglycemia in the Coronary ICU. San Diego, CA: In American Diabetes Association Scientific Sessions; 2011.

15. Haluzik M, Lips M, Drapalova J, Mraz M, Dobias M, Kopecky P, et al. The Exenatide in Patients Undergoing Coronary Artery Bypass Grafting for Improved Glucose Control and Hemodynamic Values (EXECUTIVE) Study: A Randomized. Controlled Trial. Diabetes 2014;63 Suppl 1:A261.

16. Umpierrez GE, Korytkowski M. Is incretin-based therapy ready for the care of hospitalized patients with type2 diabetes?: Insulin therapy has proven itself and is considered the mainstay of treatment. Diabetes Care 2013;36:2112-7.

17. Schwartz S, DeFronzo RA. The use of non-insulin anti-diabetic agents to improve glycemia without hypoglycemia in the hospital setting: focus on incretins. Curr Diab Rep 2014;14:466.
18. Krikorian A, Ismail-Beigi F, Moghissi ES. Comparisons of different insulin infusion protocols: a review of recent literature. Curr Opin Clin Nutr Metab Care 2010;13:198-204.

19. Umpierrez GE, Hor T, Smiley D, Temponi A, Umpierrez D, Ceron M, et al. Comparison of inpatient insulin regimens with detemir plus aspart versus neutral protamine hagedorn plus regular in medical patients with type 2 diabetes. J Clin Endocrinol Metab 2009; 94:564-9.

20. Smith WD, Winterstein AG, Johns T, Rosenberg E, Sauer BC. Causes of hyperglycemia and hypoglycemia in adult inpatients. Am J Health Syst Pharm 2005;62:714-9.

21. Maynard G, Lee J, Phillips G, Fink E, Renvall M. Improved inpatient use of basal insulin, reduced hypoglycemia, and improved glycemic control: effect of structured subcutaneous insulin orders and an insulin management algorithm. J Hosp Med 2009;4:3-15.

22. Turchin A, Matheny ME, Shubina M, Scanlon JV, Greenwood B, Pendergrass ML. Hypoglycemia and clinical outcomes in patients with diabetes hospitalized in the general ward. Diabetes Care 2009;32:1153-7.

23. Boucai L, Southern WN, Zonszein J. Hypoglycemia-associated mortality is not drug-associated but linked to comorbidities. Am J Med 2011;124:1028-35.

24. Chaney MA, Nikolov MP, Blakeman BP, Bakhos M. Attempting to maintain normoglycemia during cardiopulmonary bypass with insulin may initiate postoperative hypoglycemia. Anesth Analg 1999; 89:1091-5.

25. Nasraway SA Jr. Sitting on the horns of a dilemma: avoiding severe hypoglycemia while practicing tight glycemic control. Crit Care Med 2007;35:2435-7.

26. Gill GV, Woodward A, Casson IF, Weston PJ. Cardiac arrhythmia and nocturnal hypoglycaemia in type 1 diabetes--the 'dead in bed' syndrome revisited. Diabetologia 2009;52:42-5.

27. Tomky D. Deyection, prevention, and treatment of hypoglycemia in the hospital. Diabetes Exp 2005;18:39-44.

28. Mabrey ME, Barton AB, Corsino L, Freeman SB, Davis ED, Bell EL, et al. Managing hyperglycemia and diabetes in patients receiving enteral feedings: A health system approach. Hosp Pract (1995) 2015;43:74-8.

29. Davidson P, Kwiatkowski CA, Wien M. Management of Hyperglycemia and Enteral Nutrition in the Hospitalized Patient. Nutr Clin Pract 2015;30:652-9.

30. Gosmanov AR, Umpierrez GE. Management of hyperglycemia during enteral and parenteral nutrition therapy. Curr Diab Rep 2013;13:155-62.

31. McMahon MM, Nystrom E, Braunschweig C, Miles J, Compher C, American Society for Parenteral and Enteral Nutrition (A.S.P.E.N.) Board of Directors, et al. A.S.P.E.N clinical guidelines: nutrition support of adult patients with hyperglycemia. JPEN J Parenter Enteral Nutr 2013;37:23-36.

32. Fatati G, Mirri E, Del Tosto S, Palazzi M, Vendetti AL, Mattei R, et al. Use of insulin glargine in patients with hyperglycaemia receiving artificial nutrition. Acta Diabetol 2005;42:182-6.

33. Cook A, Burkitt D, McDonald L, Sublett L. Evaluation of glycemic control using NPH insulin sliding scale versus insulin aspart sliding scale in continuously tube-fed patients. Nutr Clin Pract 2009;24:718-22.

34. Hsia E, Seggelke SA, Gibbs J, Rasouli N, Draznin B. Comparison of 70/30 biphasic insulin with glargine/lispro regimen in non-critically ill diabetic patients on continuous enteral nutrition therapy. Nutr Clin Pract 2011;26:714-7.

35. Umpierrez GE. Basal versus sliding-scale regular insulin in hospitalized patients with hyperglycemia during enteral nutrition therapy. Diabetes Care 2009;32:751-3.

36. Korytkowski MT. In-patient management of diabetes: Controversies and guidelines. Indian J Endocrinol Metab 2013;17(Suppl 3):S630-5.

37. Sarkisian S, Fenton TR, Shaheen AA, Raman M. Parenteral nutritionassociated hyperglycemia in noncritically ill inpatients is associated with higher mortality. Can J Gastroenterol 2010;24:453-7.

38. Jacobi J, Bircher N, Krinsley J, Agus M, Braithwaite SS, Deutschman C, et al. Guidelines for the use of an insulin infusion for the management of hyperglycemia in critically ill patients. Crit Care Med 2012;40:3251-76. 
39. Neff K, Donegan D, MacMahon J, O’Hanlon C, Keane N, Agha A, et al. Management of parenteral nutrition associated hyperglycaemia: a comparison of subcutaneous and intravenous insulin regimen. Ir Med J 2014;107:141-3.

40. Roehl KA, Lach K, Coltman AE, Bacon CA, Singh S, Peterson SJ, et al. Predictors of insulin requirements among hospitalized adults receiving parenteral nutrition. JPEN J Parenter Enteral Nutr 2013;37:755-62.

41. Oghazian MB, Javadi MR, Radfar M, Torkamandi H, Sadeghi M, Hayatshahi A, et al. Effectiveness of regular versus glargine insulin in stable critical care patients receiving parenteral nutriion: a randomized controlled trial. Pharmacotherapy 2015;35:148-57.

42. Hakeam HA, Mulia HA, Azzam A, Amin T. Glargine Insulin Use Versus Continuous Regular Insulin in Diabetic Surgical Noncritically Ill Patients Receiving Parenteral Nutrition: Randomized Controlled Study. JPEN J Parenter Enteral Nutr 2016; pii:0148607116644710.

43. Baldwin D, Apel J. Management of hyperglycemia in hospitalized patients with renal insufficiency or steroid-induced diabetes. Curr Diab Rep 2013;13:114-20.

44. Gosmanov AR, Goorha S, Stelts S, Peng L, Umpierrez GE. Management of hyperglycemia in diabetic patients with hematologic malignancies during dexamethasone therapy. Endocr Pract 2013;19:231-5.

45. Spanakis EK, Shah N, Malhotra K, Kemmerer T, Yeh HC, Golden SH. Insulin requirements in non-critically ill hospitalized patients with diabetes and steroid-induced hyperglycemia. Hosp Pract (1995) 2014;42:23-30.

46. Dhital SM, Shenker Y, Meredith M, Davis DB. A retrospective study comparing neutral protamine hagedorn insulin with glargine as basal therapy in prednisone-associated diabetes mellitus in hospitalized patients. Endocr Pract 2012;18:712-9.
47. Ruiz de Adana MS, Colomo N, Maldonado-Araque C, Fontalba MI, Linares F, García-Torres F, et al. Randomized clinical trial of the efficacy and safety of insulin glargine vs. NPH insulin as basal insulin for the treatment of glucocorticoid induced hyperglycemia using continuous glucose monitoring in hospitalized patients with type 2 diabetes and respiratory disease. Diabetes Res Clin Pract 2015;110:158-65.

48. Kwon S, Thompson R, Dellinger P, Yanez D, Farrohki E, Flum D. Importance of perioperative glycemic control in general surgery: a report from the Surgical Care and Outcomes Assessment Program. Ann Surg 2013;257:8-14.

49. Frisch A, Chandra P, Smiley D, Peng L, Rizzo M, Gatcliffe C, et al. Prevalence and clinical outcome of hyperglycemia in the perioperative period in noncardiac surgery. Diabetes Care 2010; 33:1783-8.

50. Joshi GP, Chung F, Vann MA, Ahmad S, Gan TJ, Goulson DT, et al. Society for Ambulatory Anesthesia consensus statement on perioperative blood glucose management in diabetic patients undergoing ambulatory surgery. Anesth Analg 2010;111:1378-87.

51. Lazar HL, McDonnell M, Chipkin SR, Furnary AP, Engelman RM, Sadhu AR, et al. The Society of Thoracic Surgeons practice guideline series: Blood glucose management during adult cardiac surgery. Ann Thorac Surg 2009;87:663-9.

52. Umpierrez GE, Smiley D, Jacobs S, Peng L, Temponi A, Mulligan P, et al. Randomized study of basal-bolus insulin therapy in the inpatient management of patients with type 2 diabetes undergoing general surgery (RABBIT 2 surgery). Diabetes Care 2011;34:256-61.

How to cite this article: Marín-Peñalver JJ, Martín-Timón I, Javier del Cañizo-Gómez F. Management of hospitalized type 2 diabetes mellitus patients. J Transl Intern Med 2016; 4: 155-61. 